\title{
High voltage electron cooling in ion colliders
}

\section{Maxim Bryzgunov ${ }^{1}$}

1) Budker Institute of Nuclear Physics

11, Akademika Lavrentieva prospect, Novosibirsk, 630090, Russia

2) FSBI SSC RF ITEP"of NRC Kurchatov Institute"

25, Bolshaya Cheremushkinskaya street, Moscow, 117218, Russia

E-mail: M.I.Bryzgunoveinp.nsk.su

\section{Vasily Parkhomchuk}

1) Budker Institute of Nuclear Physics

11, Akademika Lavrentieva prospect, Novosibirsk, 630090, Russia

2) Novosibirsk State University

2 Pirogova street, Novosibirsk, 630090, Russia

E-mail: V.V.Parkhomchukeinp.nsk.su

\section{Vladimir Reva}

3) Budker Institute of Nuclear Physics

11, Akademika Lavrentieva prospect, Novosibirsk, 630090, Russia

4) Novosibirsk State University

2 Pirogova street, Novosibirsk, 630090, Russia

E-mail: V.B.Revalinp.nsk.su

Results of calculations of high voltage electron cooling process in NICA collider are presented. Two independent electron cooling systems in the collider are intended to increase luminosity in space charge dominated regime. The model of cooling calculations was tested on experimental results, obtained in COSY synchrotron, which is equipped with $2 \mathrm{MeV}$ electron cooler, produced by Budker INP. Also a project of high voltage electron cooling systems for the NICA collider is presented.

38th International Conference on High Energy Physics

3-10 August 2016

Chicago, USA

\section{${ }^{1}$ Speaker}




\section{Introduction}

The Nuclotron-based Ion Collider fAcility (NICA) is now under construction in the Joint Institute of Nuclear Physics (Dubna, Russia). The goal of the complex is to provide investigations in particle physics in not available before region of parameters and conditions.

In the project of the NICA a several cooling systems will be used. A booster ring will be equipped with low energy electron cooler for electron energy up to $60 \mathrm{keV}$, which will provide cooling on injection energy for beam storage and on medium energy to prepare the beam for extraction from the booster.

The collider will be equipped with both stochastic and electron cooling systems. The electron cooler maximum energy is $2.5 \mathrm{MeV}$. Coolers of such energy (higher then $1 \mathrm{MeV}$ ) usually are called "high voltage coolers". Parameters of the NICA collider electron cooling system are close to the $2 \mathrm{MeV}$ system built in Budker INP for the COSY synchrotron (Juelich, Germany). The system is a first high voltage electron cooler which utilizes, so cold, "magnetized cooling".

In the article results of electron cooling simulation for NICA collider are discussed. The simulations are based on experimental investigations of high voltage electron cooling of protons in the COSY synchrotron.

\section{High voltage electron cooler for COSY}

High voltage electron cooling system for COSY is intended to compensate heating effects in ion beam during experiments with internal target [1]. Before the installation of the high voltage electron cooler the synchrotron was equipped with cooler for electron energy up to $100 \mathrm{keV}$ [2], which provides cooling on injection energy and in low energy experiments. But to use electron cooling in whole energy range (from $25 \mathrm{keV}$ to $2 \mathrm{MeV}$ ) a new cooler was needed.

In fig. 1 a 3-D model of the high voltage electron cooling system for COSY is shown.

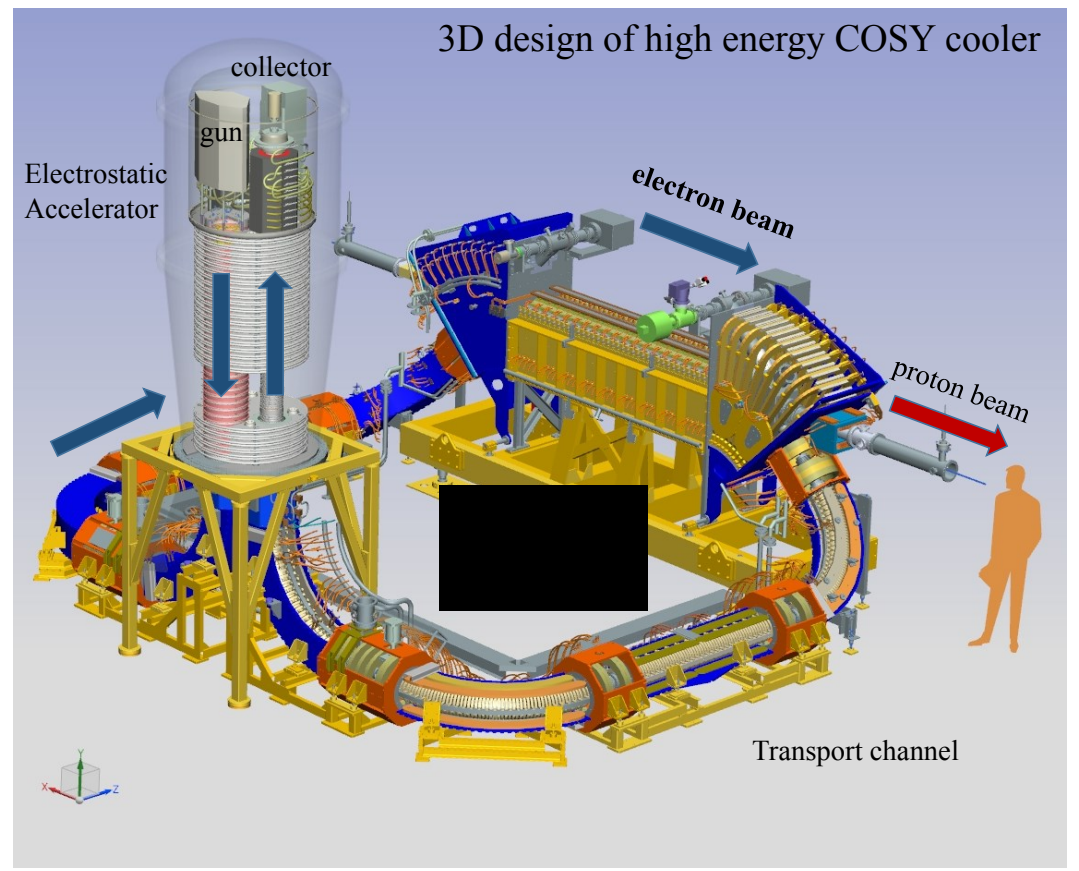

Fig. 1: 3-D view of the $2 \mathrm{MeV}$ electron cooling system for COSY 
Electron beam appears in the gun, placed in high voltage terminal in the vessel, and goes through the electrostatic tube where it is accelerated to the working energy. After acceleration, through the transport channel, it goes to cooing section where it interacts with ion beam. After the cooling section the beam goes back to the vessel, where it is decelerated by another electrostatic tube and utilized in the electron collector. Maximum electron current in the cooler is $3 \mathrm{~A}$, cathode diameter $-30 \mathrm{~mm}$, cooling section length $-2.69 \mathrm{~m}$, toroid radius $-1.0 \mathrm{~m}$, magnetic field in the cooling section $-0.5 \div 2 \mathrm{kG}$.

Strict limitations on the cooler size, related with necessity to install the cooler in existing building, leads to such a complicated shape of the cooler: after the vessel the beam goes down and then goes up to enter the cooling section.

\section{Cooling simulations for COSY}

For simulations of electron cooling a new code was developed. In the code three effects were taken into account: electron cooling, intra-beam scattering and space charge effect. The cooling force was simulated with the help of Parkhomchuk formula [3]

$$
F=-\frac{4 Z^{2} e^{4} n_{e} V}{m\left(V^{2}+V_{e f f}^{2}\right)^{3 / 2}} \ln \left(\frac{\rho_{\text {max }}+\rho_{\text {min }}+\rho_{L}}{\rho_{\text {min }}+\rho_{L}}\right),
$$

where $\rho_{\min }=Z r_{e} /(V / c)^{2}, \rho_{L}=m c V_{e T} /(e B), \rho_{\max }=V \tau /\left(1+\omega_{e} \tau\right), \tau$ is time of flight in cooling section in the beam's frame.

In order to check the code the results of simulations and experimental results of cooling process obtained in COSY on electron energy $908 \mathrm{keV}$ are shown in Fig. 2. On the left plot results of measurements of longitudinal proton distributions in bunch are shown. Black curve corresponds to initial distribution and red curve corresponds to distribution after cooling. On the right plot results of simulations are shown. One can see that results are similar but there is a difference in tails of final distribution. It can be explained by Touschek effect (scattering on big angles) which is not included in the simulations, or by not optimal coincidence of the proton and electron beam that results in very slow cooling of some part of ion beam.

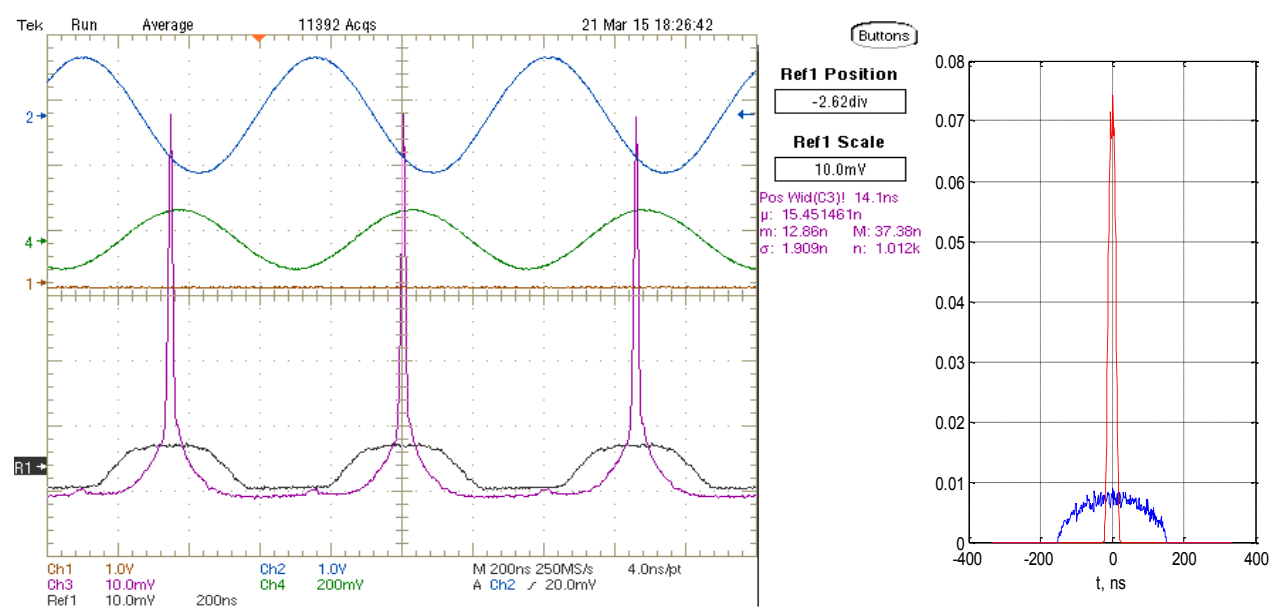

Fig. 2: Electron cooling of bunched proton beam in COSY. Electron energy $908 \mathrm{keV}$. Left result of measurements, right - results of calculations. 
In fig. 3 longitudinal momentum spread for the same experiment and results of calculations are shown.
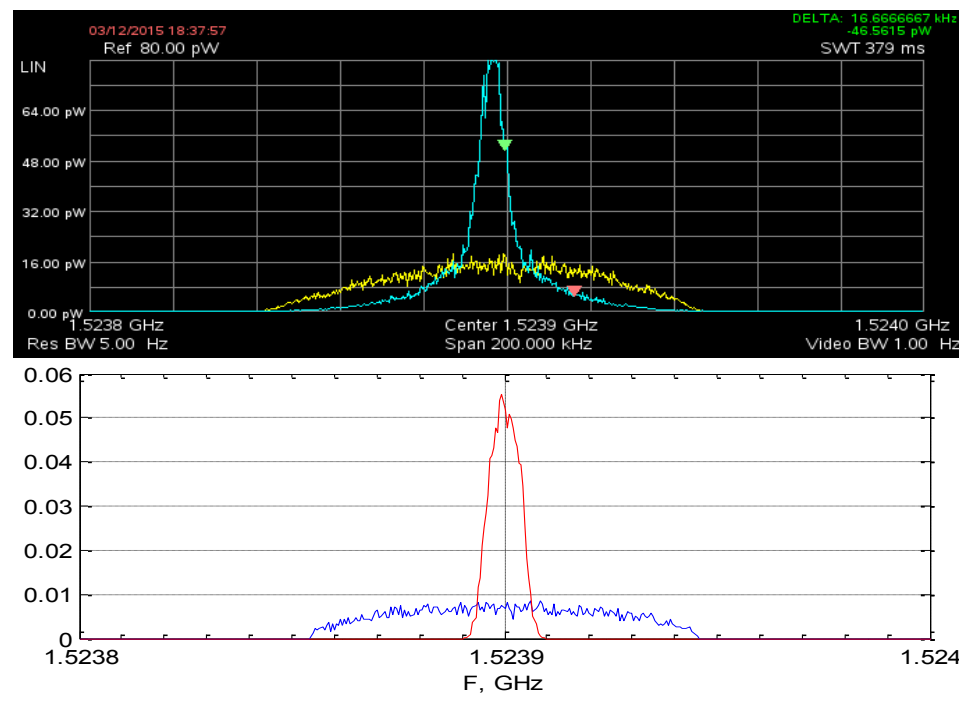

Fig. 3: Upper - Schottky signal for the non-cooled (yellow) and cooled (blue) states of the proton beam. Lower - result of the simulation.

Comparing the plots one can see the same difference in tails of distributions as in fig. 2 , which can be explaned by by Touschek effect.

Comparison of simulation result with measurements shows that the code is good enough for calculations of electron cooling in high voltage systems.

\section{Cooling simulations for NICA collider}

In fig. 4 results of longitudinal cooling simulations for bunch in NICA collider are shown. The bunch initially ocupies hulf of separatrix. After $160 \mathrm{sec}$ the distribution comes to equilibrium state and cooling is over. In this case the bunch length is determined by its space charge. The bunch contains $2.3 \cdot 10^{9}{ }^{197} \mathrm{Au}^{79+}$ ions with energy $4.5 \mathrm{GeV} / \mathrm{u}$. Electron current is $1 \mathrm{~A}$, RF amplitude - 0.6 MV, harmonic number -66 , cooling section length $-6 \mathrm{~m}$, magnetic filed in the cooling section $-2 \mathrm{kG}$, ring perimeter $-503 \mathrm{~m}$. For simulations $10^{4}$ test particle were used.
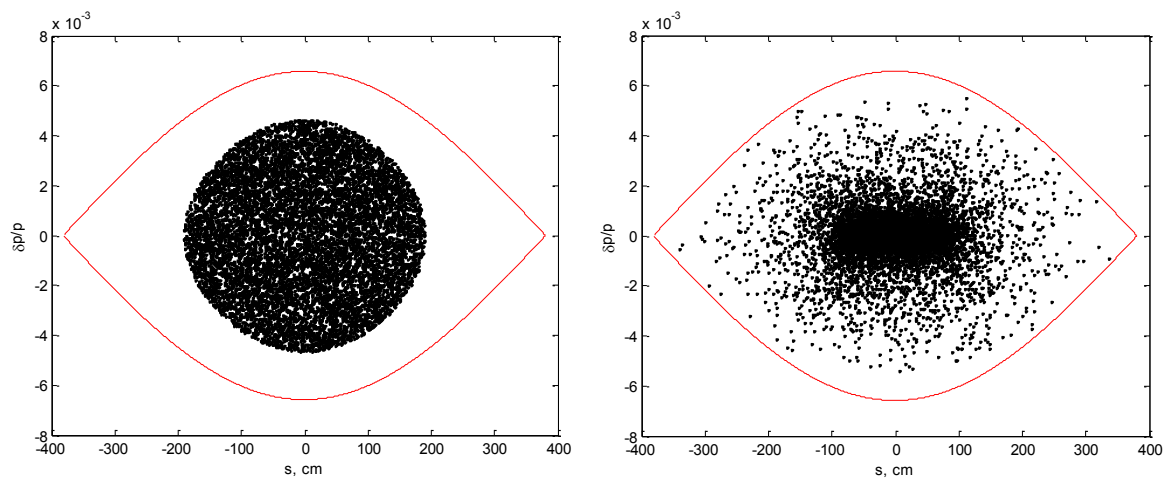

Fig. 4: longitudinal phase space plot for electron cooling in NICA collider. Left - before cooling, right - after $160 \mathrm{sec}$. 
In fig. 5 longitudinal spatial and momentum distribution before cooling and after $160 \mathrm{sec}$ are shown.
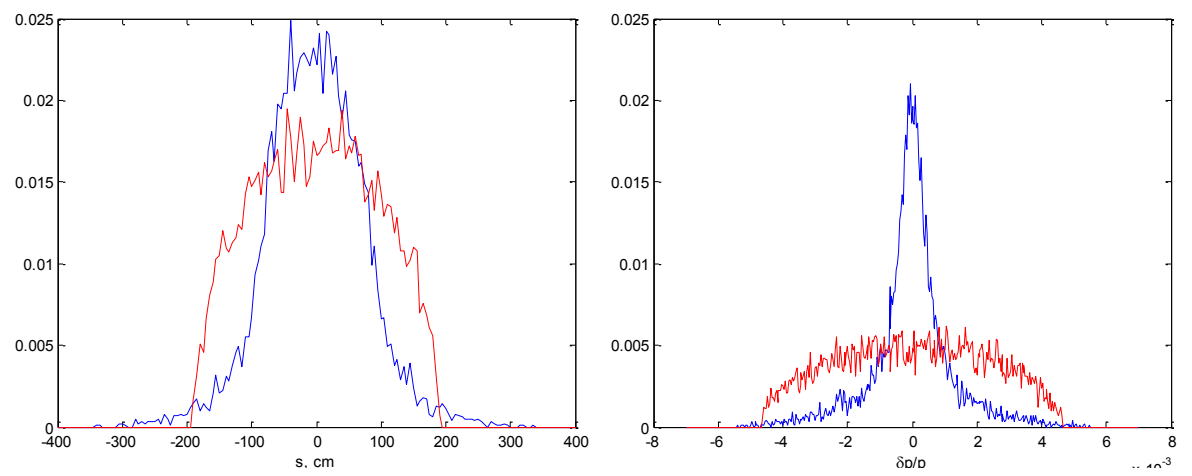

Fig. 5: Longitudinal spatial (right) and momentum (left) distributions before cooling (red) and after $160 \mathrm{sec}$ (blue).

High ion density leads to high IBS rate. Because of this there are particles which go out from core of the distribution and form tails.

Simulation results show that electron cooling in the NICA collider is effective way to decrease beam emittance. Feature of the electron cooling kinetics is that increase of beam energy relative to COSY experiment significantly decreases cooling force, but high charge of ions in the NICA collider increases the force to high enough values.

\section{Construction of the NICA high voltage electron cooler}

Since cooling of beams should be provided during beam collision permanently, the electron cooling system must cool both beams simultaneously. It means that there must be two cooling sections with electron beams moving in opposite directions and that the system must contain two independent or almost independent electron coolers.

In fig. 63 -D view of the system, proposed by Budker INP is shown. The construction of each cooler is based on COSY cooler and contains high voltage vessel, transport channels and cooling section.
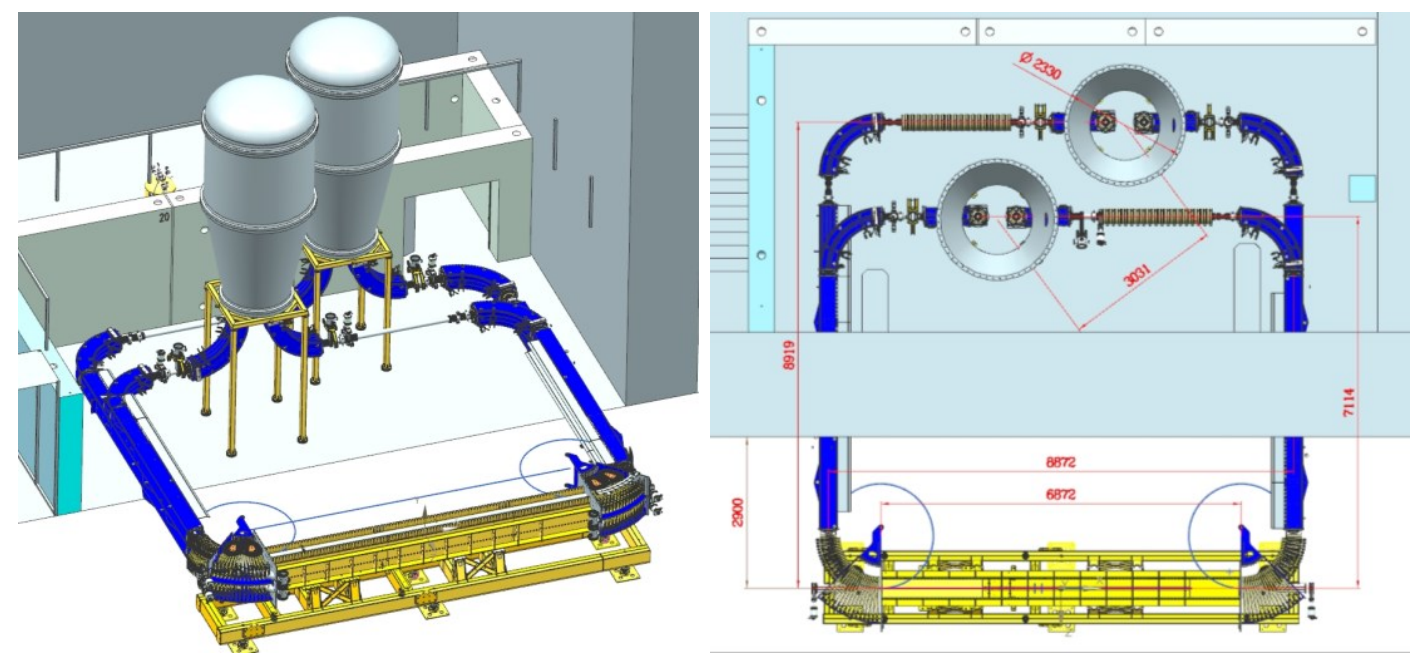

Fig. 6: 3-D view of the electron cooling system for the NICA collider. 
Distance between ion beams is $32 \mathrm{~cm}$. In order to preserve place for cooling it was decided to place two cooling section one above the another. It produces big technical troubles, but allow us to make cooling sections length about $6 \mathrm{~m}$ for each beam.

On whole trajectory from a gun to a collector electrons move in guiding magnetic field. It allows to focus the beam in transport channels and to avoid problems related with entry to magnetic field of a cooling section, because the field there is necessary to make, so called, "magnetized cooling".

High voltage vessels of the systems work independently that allows adjust energy of electron beams separately in order to reach maximum cooling efficiency for both ion beams. As in COSY cooler high voltage systems are produced with modular principle and powered with the help of cascade transformer [4].

\section{Conclusion}

Electron cooling for the NICA collider is an effective way to increase luminosity by decreasing beam emittances. Increasing of electron cooling energy decreases its efficiency, but high charge of ions in the NICA collider increase cooling force to high enough values.

Experience of the Budker INP in electro cooling, especially achieved during commissioning of $2 \mathrm{MeV}$ electron cooling system for the COSY synchrotron allows to expect that the system for the NICA collider, which is designed now, will provide needed cooling parameters.

\section{Acknowledgements}

Authors would like to thank V. Gosteev form the BINP electron cooling team for the help during preparations of the article.

\section{References}

[1] Dietrich J., Parkhomchuk V., Reva V. et al, The proposed $2 \mathrm{MeV}$ electron cooler for COSY, in proceedings of COOL'05 workshop, 299.

[2] Derissen W., Maier R., Pfister U. et al, Status of the COSY Electron Cooler, in proceedings of EPAC'92 conference, 839 .

[3] Parkhomchuk V.V., New insights in the theory of electron cooling, NIM A 441 (2000) 9.

[4] Skorobogatov D., Bryzgunov M., Goncharov A., et al, The power supply system for the accelerating column of the $2 \mathrm{MeV}$ electron cooler for COSY, in proceedings of RuPAC'12 conference, 512. 